\title{
Swim Training Initiated Acutely after Spinal Cord Injury Is Ineffective and Induces Extravasation In and Around the Epicenter
}

\author{
Rebecca R. Smith,, Edward H. Brown,, Alice Shum-Siu, Ashley Whelan,, Darlene A. Burke, \\ Richard L. Benton, ${ }^{1,2}$ and David S.K. Magnuson ${ }^{1,2}$
}

\begin{abstract}
Activity-based rehabilitation is a promising strategy for improving functional recovery following spinal cord injury (SCI). While results from both clinical and animal studies have shown that a variety of approaches can be effective, debate still exists regarding the optimal post-injury period to apply rehabilitation. We recently demonstrated that rats with moderately severe thoracic contusive SCI can be re-trained to swim when training is initiated 2 weeks after injury and that swim training had no effect on the recovery of overground locomotion. We concluded that swim training is a task-specific model of post-SCI activity-based rehabilitation. In the present study, we ask if re-training initiated acutely is more or less effective than when initiated at 2 weeks post-injury. Using the Louisville Swim Scale, an 18-point swimming assessment, supplemented by kinematic assessment of hindlimb movement during swimming, we report that acute re-training is less effective than training initiated at 2 weeks. Using the bioluminescent protein luciferase as a blood-borne macromolecular marker, we also show a significant increase in extravasation in and around the site of SCI following only $8 \mathrm{~min}$ of swimming at 3 days post-injury. Taken together, these results suggest that acute re-training in a rat model of SCI may compromise rehabilitation efforts via mechanisms that may involve one or more secondary injury cascades, including acute spinal microvascular dysfunction.
\end{abstract}

Key words: activity-based rehabilitation; microvascular; rat; spinal cord injury; swimming

\section{Introduction}

$\mathrm{O}$ NE OF THE MOST PROMISING THERAPEUTIC APPROACHES to the treatment of spinal cord injury (SCI) is body weightsupported step training (BWST) (Harkema et al., 1997; BeresJones et al., 2003). BWST is a specific form of activity-based rehabilitation that seeks to re-train the spinal cord to generate functional stepping by exposing it to the specific pattern of afferent input associated with stepping, with assistance, on a treadmill. While the majority of clinical studies using BWST focus on chronic SCI (Pepin et al., 2003; Maegele et al., 2002; Abel et al., 2002; Gazzani et al., 1999; Felici et al., 1997; Harkema et al., 1997), a number of animal studies suggest that activity-based rehabilitation strategies may be more effective when initiated acutely, and that the benefits may go beyond locomotion to include pain, spasticity, muscle wasting and cardiovascular/respiratory function. For example, significant benefits were found for early "exercise" (Dupont-Versteegden et al., 2004; Engesser-Cesar et al., 2007), and acute re-training on a treadmill appears to have significant advantages over training in chronic rodent models (Gomez-Pinella et al., 2001, 2002; Hutchinson et al., 2004). Further, studies utilizing the completely transected cat model also demonstrate enhanced efficacy of rehabilitation initiated within days of injury (Boyce et al., 2007; Rossignol, 2002; DeLeon et al., 1998). Likewise, Multon et al. (2003) showed that rats that began treadmill training as early as 2-4 days after a compressive SCI had higher Basso, Beattie, and Bresnahan (BBB) scores compared to untrained animals. These data collectively support the concept that initiating training acutely following an incomplete SCI may enable activation of spinal circuitry during a period when the perceived plasticity in the system is high.

\footnotetext{
${ }^{1}$ Kentucky Spinal Cord Injury Research Center, Department of Neurological Surgery, Louisville, Kentucky.

${ }^{2}$ Department of Anatomical Sciences and Neurobiology, University of Louisville School of Medicine, Louisville, Kentucky.
} 
However, contusive injuries to the brain and spinal cord involve secondary injury cascades that encompass the acute and sub-acute post-injury time period and thus may complicate the optimization of the timing of acute rehabilitative intervention. For example, in a series of studies using the fluid percussion model of traumatic brain injury (TBI) Greisbach et al. (2004a,b, 2007) have shown that voluntary exercise within the first 7-10 days post-injury may be detrimental to functional outcome. While these data show that voluntary exercise can induce increases in growth factor expression and improved functional outcome, these beneficial effects were compromised if initiated within the first 7-10 days postinjury. The poor functional outcomes were accompanied by augmented epicenter histopathology, which the authors suggest may be correlated with a compromised blood-brain barrier. Whether or not similar principles apply to SCI is currently not known.

Many events occurring over the first several days following a contusive SCI are believed to be crucial to the expanding lesion and secondary injury processes, and are therefore potential targets for therapeutic intervention. These include the breakdown of the blood-spinal cord barrier (BSCB) (Whetstone et al., 2003), which allows the infiltration of both cellular and non-cellular inflammatory mediators. The temporal course of permeability changes to the BSCB following contusive SCI has been demonstrated in the rat by Popovich et al. (1996). Their data show that permeability at or near the injury epicenter is maximal at 3 days post-injury and concentrated within affected gray matter. The breakdown of the BSCB begins to resolve by 7 days, but is still not equivalent to uninjured spinal cord at 14 days post-injury. More recently, this temporal course of BSCB compromise and reconstitution has also been observed following contusive SCI in the mouse (Whetstone et al., 2003). Importantly, this temporal progression of known BSCB instability encompasses the acute and sub-acute time periods when therapeutic strategies, including activity-based rehabilitation, could realistically be initiated.

We showed previously that adult female Sprague-Dawley rats can be re-trained to swim after a moderately severe thoracic contusion injury, when the animals are re-introduced to the water at 10 days, and rigorously re-trained (i.e., $6 \times 4$-min swim sessions 4 times per week over 8 weeks). This intervention resulted in significantly improved swimming as assessed by the Louisville Swim Scale (LSS) (Smith et al., 2006b) but did not translate to enhanced recovery of overground locomotion as assessed by the BBB Open Field Locomotor Scale (Basso et al., 1995). These observations lead us to conclude that swim-training, when applied in the sub-acute phase of SCI recovery, is a task-specific model of activitybased rehabilitation, which could be used to investigate postSCI plasticity, independent of over-ground locomotion. Common to all activity-based rehabilitation approaches is the fact that activity (exercise) induces increases in blood flow, heart rate, and respiration rate. How the stresses associated with physical activity early after SCI alters the cascade of secondary injury events and the microvasculature surrounding an injury epicenter is essentially unknown. Thus, the primary goals of the current study were to examine the influence of swim-training initiated acutely (3 days) post-injury on the recovery of swimming and, subsequently, to investigate the effects of swim-training sessions on the evolving microvascular plasticity in the injured spinal cord.

\section{Methods}

\section{Experimental overview}

The experiments reported were completed in three phases according to the timelines shown in Figure. 1. The initial experiment (Phase 1) was designed to investigate rehabilitative effects of acute swim training. A secondary finding of this study was that the control animals, which were assessed in the swimming pool twice each week, showed a significant improvement in swimming over time, suggesting that $8 \mathrm{~min}$ of swimming each week was sufficient to induce a training effect. This question was addressed in Phase 2, where animals were also trained acutely and assessed weekly; however, an untrained control group was only assessed terminally (Fig. 1). Finally, based on the findings of Phases 1 and 2, a third study (Phase 3 ) was completed where animals were exposed to $2 \times 4$ min training sessions, or cage rest, at 3 or 14 days post-SCI, with each animal assessed for overt signs of microvascular dysfunction (i.e., BSCB permeability).

\section{Pool design and baseline measurements}

All the animals were acclimatized to the training pool 1 week before surgery. They were allowed to swim several laps in the pool each day for 5 consecutive days before surgery. The acclimatization period allowed the uninjured animals to become comfortable with the handler and the pool, and allowed the trainers to observe whether any pre-injury deficits were present. The pool is a clear Plexiglas tank 60 inches long, 7 inches wide, and 12 inches deep. The pool was filled to a depth of 7-8 inches for the training sessions, and the water temperature was maintained at $25-28^{\circ} \mathrm{C}$. All swim training for these studies included supplementary cutaneous feedback, in the form of suspended 1.5-ml centrifuge tubes, as described previously (Smith et al., 2006a,b). The centrifuge tubes provided cutaneous feedback to the hindpaws during the training session, but offered less than $1.2 \mathrm{~g}$ of buoyancy each. One end of the pool has an exit ramp covered with a neoprene pad, which allowed the animals to exit the pool easily, even after injury. Baseline LSS, hindlimb kinematics during swimming, and BBB Open Field Locomotor scores were obtained prior to injury (Smith et al., 2006b; Basso et al., 1995).

\section{Spinal cord injury}

Twelve young adult female Sprague-Dawley rats (175$200 \mathrm{~g}$ ) were used for each of the acute training studies (Phases 1 and 2). The rats were anesthetized with Nembutal $(0.8 \mathrm{cc} / 100 \mathrm{~g}$, ip) and were given prophylactic antibiotics (Gentamicin, $15 \mathrm{mg} / \mathrm{kg}$, sc). A single level laminectomy was performed at the T9 vertebra, and the spine was immobilized using clamps applied to the T8 and T10 spinous processes. An NYU Impactor was used to deliver a $25 \mathrm{~g}$-cm contusion injury at the T9/10 spinal level. After the injury, the wound was closed in layers and a topical antibiotic ointment applied to the incision. The animals were placed on heating pads in clean, single cages overnight, and returned to their regular housing room the following day. Bladder expression was performed twice daily until adequate spontaneous voiding occurred. Once training commenced (3 days post-injury), animals were housed two per cage for the duration of the study. In Phase 1, two animals were removed based on injury 


\section{Phase 1}

Trained Group

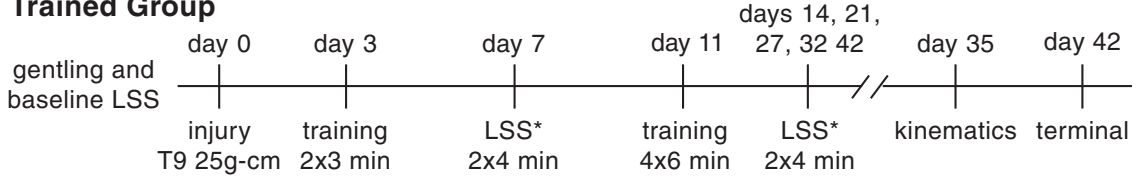

\section{Control Group}

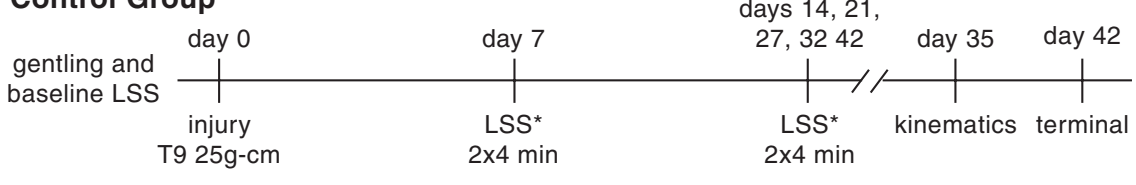

*LSS 4 minutes each with and without cutaneous feedback.

\section{Phase 2}

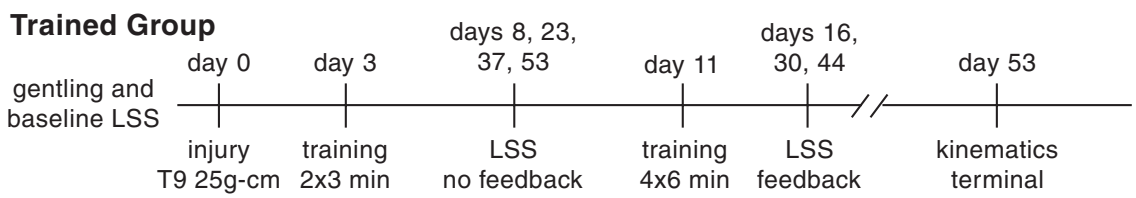

\section{Control Group}

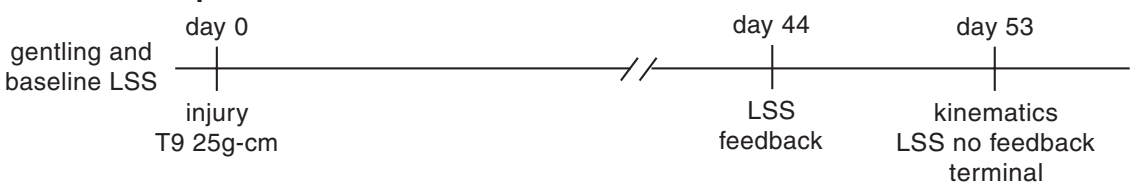

Phase 3
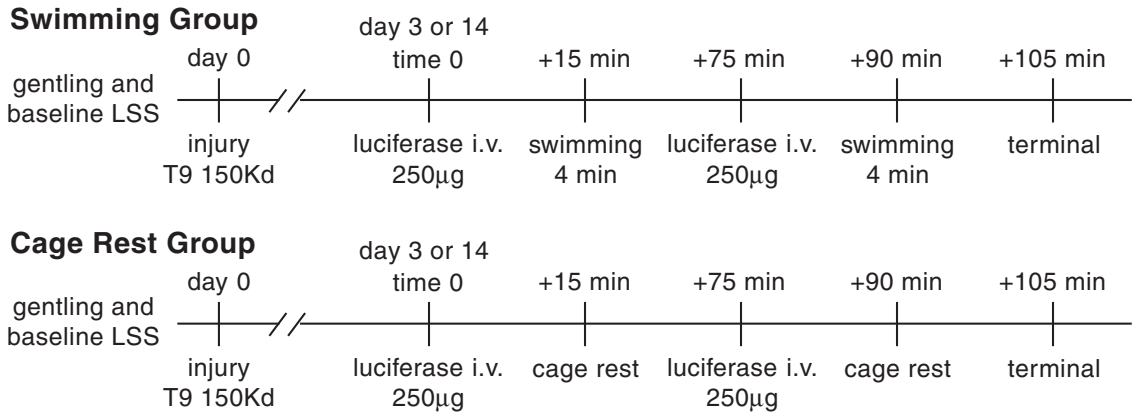

FIG. 1. Shown are timelines for all three experiments. In Phase 1, both groups of animals were assessed using the Louisville Swim Scale (LSS) twice weekly, once with and once without cutaneous feedback. In Phase 2, the trained group was assessed once weekly, alternating between with and without cutaneous feedback. The control group was assessed only twice, on day 44 with cutaneous feedback and on day 53 without feedback, during which video was made for kinematic assessment. Injuries for Phases 1 and 2 were moderately severe, delivered by the New York University (NYU) Impactor. Injuries for Phase 3 were moderate, delivered by the Infinite Horizons (IH) contusion device.

severity (displacement and/or velocity of impact), and one animal died after 4 weeks of training, leaving final group sizes of five (swim-trained) and four (untrained). In Phase 2, one animal died within 3 days of injury, and two others were removed based on injury severity (displacement and/or velocity of impact), leaving final group sizes of five (swimtrained) and four (untrained). An additional 48 young female Sprague-Dawley rats were used to assess spinal microvascular permeability (Phase 3). All procedures were identical to those described above, except that the Infinite Horizons Impactor was used to deliver a moderate (150-kD) contusion injury.

\section{Phases 1 and 2: Acute post-injury swim training}

For the Phase 1 study, the animals in the trained group were introduced to the pool for $2 \times 3$-min training sessions beginning on post-injury day 3 and were assisted by handlers when necessary. This was increased to $4 \times 3$-min sessions on days 4 and 5. Starting on day 8 , the duration of each session was increased to $4 \mathrm{~min}$, and by day 11 , each animal was training for $6 \times 4$-min sessions daily, for 4 days each week (Fig. 1). Untrained animals for this study remained caged (in pairs) in the swimming room during the training sessions. Untrained animals were handled daily and received sweetened 
cereal treats, with the trained animals, at the completion of each training session. This study continued for 6 weeks.

Training for Phase 2 was identical to Phase 1, however, this study continued for 7 weeks. As with Phase 1, untrained animals remained caged (in pairs) in the swimming room during the training sessions, were handled daily, and received cereal treats with the trained animals.

\section{Phases 1 and 2: Weekly assessments}

The BBB Open Field Locomotor Scale (Basso et al., 1995) was used to score overground locomotion of all animals on a weekly basis starting at 1 week post-injury, as previously described (Smith et al., 2006a,b). In addition, swimming function was assessed using the LSS (Smith et al., 2006b), which sensitively quantifies swimming ability of SCI rats utilizing an 18-point scale with three functional ranges: 0-5 (poor swimmer), 6-11 (intermediate swimmer), and 12-17 (good swimmer). Within the scale, hindlimb movement is assessed as absent ( $<5 \%$ of the 4 -min assessment time), occasional $(6-50 \%)$, frequent (51-95\% of the time), or consistent $(>95 \%)$. For Phase 1, the trained and untrained animals were tested weekly both with and without supplemental cutaneous feedback, making the total testing time 8 min per week. For Phase 2, the trained animals were assessed weekly (4 min/ week). Testing was conducted with supplemental cutaneous feedback on weeks 2,4 , and 6 and without feedback on weeks $1,3,5$, and 7 . The untrained animals were not tested at all until the last 2 weeks of the study. At this point, they were tested with feedback on week 6 and without on week 7 (Fig. 1).

\section{Phases 1 and 2: Swimming kinematics}

One drawback of the LSS is that it scores hindlimb movement and alternation, but does not distinguish between small, ineffective movements or larger, more effective swimming strokes. To quantitatively assess swimming movements, kinematic analysis was done of the nine best swimming strokes taken from three different passes (pool lengths) both preinjury and at week 5. This analysis represents the best movements that were produced during the trials, rather than those that are consistently produced.

Before injury, each rat was shaved on the left side so that the iliac crest and hip could be easily palpated. A green tattoo was placed on the skin immediately overlying the iliac crest and greater trochanter of the femur (hip joint) using an Aramis micro-tattoo punch (Braintree Scientific, Braintree, MA). Before each filming session, the animal was shaved and a black Sharpie pen was used to reinforce the original tattoo marks on the iliac crest and hip and to place additional marks on the ankle (lateral malleolus) and toe (metatarsophylangeal joint). Three complete swimming passes (pool lengths) where the hindlimbs remained vertical were recorded with a highspeed $(60-\mathrm{Hz})$ Basler camera and saved as AVI files. These files were opened in MaxTraq (Innovation Systems, Columbiaville, MI), where the iliac crest (I), hip (H), ankle (A), and toe $(\mathrm{T})$ were marked in a semi-automated fashion on each frame of the digital video. Each pass was then divided into strokes based on the peak extension of the hip-ankle-toe (HAT) angle and an angle-angle plot (iliac crest-hip-ankle [IHA] vs. HAT) was constructed for each stroke. An angleangle plot of a normal stroke forms an ellipse, with the area representing the out-of-phase excursions of the proximal and distal angles and the center of the ellipse (the centroid) representing the mean of each angle taken from all the points used to create the plot. The area and centroid of each ellipse was calculated using an Elliptical Fourier Analysis freeware application (Ferson et al., 1985). Thus, each stroke was objectively characterized using the ellipse area and the $x$ - and $y$-values of the centroid. If the animals were unable to kick, the IHA/HAT values were broken into virtual strokes of 15 frames each and analyzed in the same way.

\section{Phases 1 and 2: Histologic assessment}

After the terminal assessments, the animals were euthanized with an excess of anesthetic (Nembutal, $0.3 \mathrm{cc}$, ip) and perfused transcardially with calcium-free tyrodes and $4 \%$ paraformaldehyde. The spinal cords were removed and postfixed overnight followed by cryoprotection in 30\% sucrose. Once the tissue was cryoprotected, a 6-mm segment of spinal cord containing the epicenter was blocked in the transverse plane, placed in molds with cutting media, frozen on dry ice and stored at $-80^{\circ} \mathrm{C}$. Spinal cord tissue was cut transversely at $30 \mu \mathrm{m}$ and mounted onto gelatinized glass slides. One set of slides was stained with Eriochrome Cyanin (Scheff et al., 2003) to enable the determination of spared white matter at the injury epicenter. Digitized images of stained sections were captured using a Spot camera (Diagnostic Instruments, Sterling Heights, MI) connected to a Nikon Eclipse E400 microscope. The images were opened in Appleworks 6.0, and the sections were traced using a Wacom Intuos drawing tablet (Vancouver, WA) and saved as uncompressed TIF files. Each TIF file was opened in Image J, and the area of the gray and white matter was calculated. The injured white matter area for each epicenter was converted to a spared white matter percentage using a mean uninjured thoracic white matter area calculation as the denominator.

\section{Phase 3: Assessment of microvascular dysfuction}

In this experiment, we examined the extravasation of the macromolecule luciferase into parenchyma of the spinal cord as a quantitative marker of BSCB dysfunction (Whetstone et al., 2003). This was done using uninjured control and SCI animals subjected to $2 \times 4$-min swimming sessions $(75 \mathrm{~min}$ apart) compared to cage-rested controls. We used six groups of six animals to compare uninjured controls with 3-day and 14-day post-injury time points. Immediately before each swimming session, $250 \mu \mathrm{l}$ of luciferase $(1 \mathrm{mg} / \mathrm{ml}$; Promega, Madison, WI) was administered intravenously via the lateral tail vein. Animals in the swim-trained group received 4 min of training starting $15 \mathrm{~min}$ after luciferase administration (Fig. 1). After the second and final swimming session, the animals were returned to their home cages for an additional $15 \mathrm{~min}$ prior to being euthanized with an excess of Nembutal $(0.3 \mathrm{cc}$, i.p.) and perfused transcardially with $250 \mathrm{ml}$ of $10 \mathrm{mM}$ phosphate-buffered saline (PBS). Un-trained rats received luciferase but remained in their home cages, singly housed. Three 4-mm segments of spinal cord tissue, one centered on the injury epicenter and adjacent rostal/caudal segments, were rapidly removed, weighed, and homogenized in $250 \mu \mathrm{l}$ of luciferase lysis buffer (Promega). As an intra-animal tissue control, a 4-mm segment was blocked from the mid-cervical spinal cord (i.e., uninjured tissue). Samples were flash-frozen on dry ice and stored at $-80^{\circ} \mathrm{C}$ until analysis. Luciferase ac- 
tivity in each experimental sample was determined using the Promega Luciferase Assay kit (no. E1501) according to the manufacturer's protocol. Reacted samples were read on a Orion microplate luminometer with Simplicity 4.0 software (Berthold Detection Systems, Oak Ridge, TN). All samples were analyzed in triplicate, independently replicated, and normalized to wet-weight.

\section{Statistical analysis}

Behavioral data (BBB, LSS, and kinematics) and quantitative permeability data were analyzed using a repeatedmeasures analysis of variance (ANOVA), followed by a Tukey HSD post-hoc $t$-test to correct for small sample size and unequal variance among groups. Spared white matter data were analyzed using a two-tailed independent $t$-test for equal or unequal variance as appropriate (Hays, 1981).

\section{Results}

\section{Over-ground locomotion}

Animals in both the Phase 1 and 2 training studies were assessed weekly for changes in over-ground locomotion using the BBB Open Field Locomotor Scale (Basso et al.,1995). The mean terminal BBB scores for all the groups ranged from 9.2 to 10.6, indicating that the animals recovered weight support at stance and occasional weight-supported plantar stepping with a lack of forelimb/hindlimb coordination. The mean BBB scores for the trained and untrained groups in the Phase 2 study were found to be statistically different, with the swimtrained animals displaying slightly poorer overground stepping as compared to the untrained group (Table 1).

\section{Spared white matter}

The spared white matter at the injury epicenter is shown as a percent of uninjured tissue in Table 1. No group differences were found. For comparison purposes, the mean spared white matter from our previously published "standard" study is also shown. Note that the spared white matter was assessed using cresyl violet stain in the standard study and eriochrome cyanin in the current studies.

\section{Swimming}

Phase 1: Acute training study. Pre-injury, all the animals in the Phase 1 study swam normally with LSS scores of 17.
Post-injury assessments were done with supplemental cutaneous feedback in the morning (Fig. 2A), and then without supplemental cutaneous feedback in the afternoon, $2 \mathrm{~h}$ later (Fig. 2B). At the first post-injury LSS assessment on day 7, after 4 days of swim training, both trained and untrained groups were assessed for $4 \mathrm{~min}$, or as long as they could safely swim. At this time point, none of the animals moved their hindlimbs, and all had severe trunk rotation and/or severe body angle, illustrating that 4 days of swim training with supplemental cutaneous feedback, initiated at 3 days post-injury, had no influence on swimming (Fig. 2). However, after this time point, both groups showed improved LSS scores over time. There were no differences in LSS scores between the two groups at any time point, and only one animal recovered either occasional (6-50\%) or frequent (51-95\%) hindlimb movement. It was a trained animal that scored 3 on day 7 and scored 11 on day 42. These findings are in contrast to our previously published study (standard study), wherein animals that were swim trained with or without cutaneous feedback starting at 2 weeks post-injury scored significantly better than untrained animals that were assessed weekly (Smith et al., 2006a). All the trained animals in the standard study recovered at least occasional hindlimb movement. For comparison, Figure 2 shows the 42-day LSS scores for groups from the standard study that were trained with (A) or without (B) cutaneous feedback.

Kinematic analysis was done to quantitatively describe hindlimb movement during swimming based on the excursions of the HAT and IHA angles. Figure 3A shows an angleangle plot (HAT vs. IHA) from one baseline stroke, as an example. The area of the ellipse represents the out-of-phase excursion of the two angles, and the centroid represents the mean position of the limb throughout the stroke cycle. For example, Figure 3B also shows a stroke produced by an untrained animal that had a very extended ankle $(x=166)$, but that still managed to generate a fairly large stroke with an angle-angle plot area of 28 .

Figure 3C shows that the mean angle-angle plot area was significantly reduced at 5 weeks in the swim-trained group as compared to baseline, but not for the untrained group. This reflects the fact that three out of four of the untrained animals recovered the ability to generate strokes with large angular excursions, compared to only one out of five of the trained animals. In addition, it suggests that only 8 min of swimming per week is sufficient to bring about some functional recovery

Table 1. BBB Open-Field Locomotor Scores and Spared White Matter (\%)

\begin{tabular}{llccrrr}
\hline Study & Train & Training & Assessment & BBB $( \pm S D)$ & $\mathrm{n}$ & SWM \% $( \pm S D)$ \\
\hline Standard $^{\mathrm{a}}$ & Swim & 10-60 dpi & Weeks 1-9 & $9.9 \pm 1.6$ & 8 & $8.6 \pm 5.3^{\mathrm{b}}$ \\
Standard $^{\mathrm{a}}$ & None & & Weeks 1-9 & $9.6 \pm 1.4$ & 7 & $8.8 \pm 6.4^{\mathrm{b}}$ \\
Phase 1 & Swim & $3-42$ & Weeks 1-6 & $9.2 \pm 1.2$ & 5 & $10.3 \pm 3.2$ \\
Phase 1 & None & & Weeks 1-6 & $9.6 \pm 1.0$ & 4 & $9.0 \pm 3.5$ \\
Phase 2 & Swim & $3-35$ & Weeks 1-5 & $9.3 \pm 0.5^{\mathrm{c}}$ & 6 & $11.5 \pm 4.3$ \\
Phase 2 & None & & Weeks 1-5 & $10.6 \pm 1.0^{\mathrm{c}}$ & 5 & $13.6 \pm 3.9$ \\
\hline
\end{tabular}

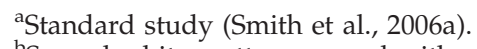

${ }^{\mathrm{b}}$ Spared white matter assessed with cresyl violet.

${ }^{\mathrm{C}}$ Unpaired t-test assuming unequal variance, $p<0.05$.

BBB Open-Field Locomotor Scale presented as mean \pm SD.

Spared white matter (SWM) presented as percent \pm SD.

Days post injury (dpi) presented as a range. 
A Swimming with supplemental cutaneous feedback

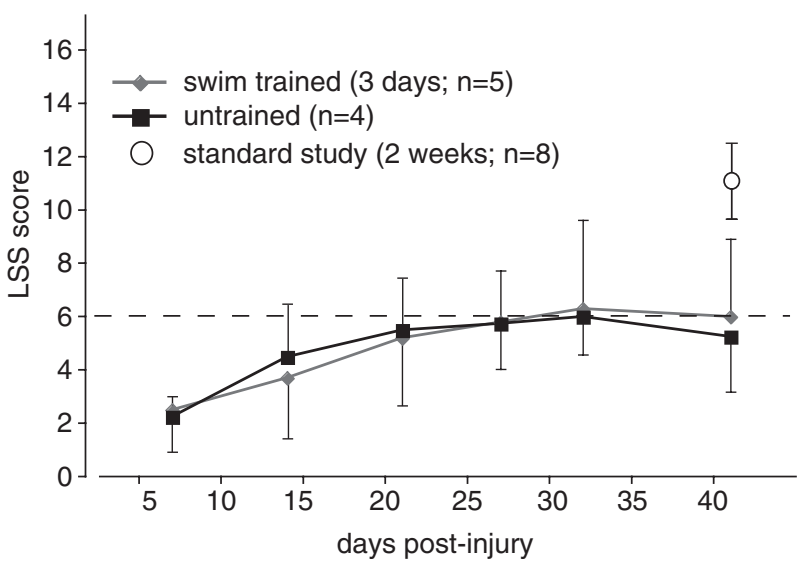

B Swimming without supplemental cutaneous feedback

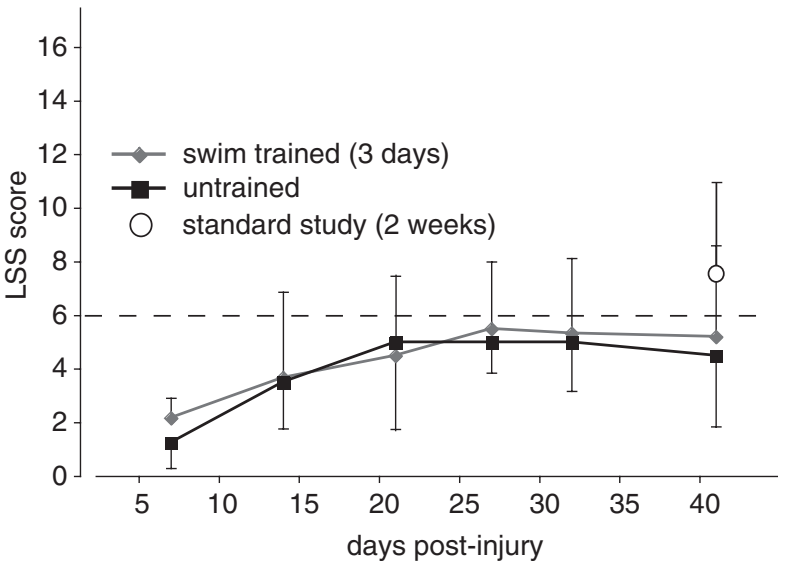

FIG. 2. Shown are the Louisville Swim Scale (LSS) scores, over time, for swim-trained and untrained animals assessed with (A) and without (B) supplementary cutaneous feedback from the Phase 1 study. For comparison purposes, the 42-day LSS scores are shown for our previously published study (Smith et al., 2006a), where animals were swim trained starting at 2 weeks post-injury. The dashed line represents an LSS score of 6, which marks the transition from low to moderate swimming and the recovery of at least occasional hindlimb movement occurring $6-50 \%$ of the 4 -min assessment period.

of hindlimb movement, including occasional swimming strokes that have a large excursion and are relatively effective. Figure 3D, showing the mean angle-angle plot centroids, indicates that both trained and untrained groups had a more extended mean limb position while swimming, and the significant $x$-shift illustrates that the distal limb segment (knee and ankle) were responsible for this difference. The large amount of variability, in particular in the HAT angle, shows that some animals swam with somewhat flexed ankles and others with ankles that were very extended (Fig. 3B), while limb position during swimming was not positively influenced by acute training.

Phase 2: Swim training and LSS assessments. In the Phase 2 study, the LSS scores of the trained animals did not show statistically significant improvements over time $(p=0.102$; Fig. 4). This is in part because only one of the five trained animals recovered occasional to frequent hindlimb movement by post-injury day 30 , resulting in high statistical variability for the group. This hindlimb movement was observed only when the animal was assessed with cutaneous feedback. None of the animals recovered even occasional hindlimb movement when assessed without cutaneous feedback (Fig. 4). Surprisingly, three out of four of the untrained animals, which were not assessed until post-injury day 44, exhibited frequent to consistent hindlimb movement $(\mathrm{LSS}=12$ ) and the fourth exhibited occasional hindlimb movement (5$50 \%$; LSS $=8$ ). The mean score of 11 is significantly higher than the mean $(6.2)$ of the trained group $(p<0.05)$. Interestingly, the terminal (day 44) LSS score for the untrained group was $11.0 \pm 2.0(n=5)$ when assessed with cutaneous feedback, which is similar to the 6-week score for the trained group from our published "standard" study $(11.3 \pm 2.3, n=8)$, where training was initiated at 2 weeks post-injury.

Terminal kinematic analysis also showed that here were no differences between the trained and untrained groups (Fig. $4 \mathrm{~B}, \mathrm{C})$. Three animals in each group were able to generate some kicks with substantial joint excursions resulting in large group standard deviations even if they could not achieve even occasional to frequent hindlimb movements during the 4-min LSS scoring session (LSS $<6$ ). Figure. $4 \mathrm{C}$ shows that the two groups also had similar mean limb positions as illustrated by the angle-angle plot centroid.

Phase 3: Augmentation of BSCB dysfunction by acute swim training. As shown in Figure 5A, we found that only 8 min of swim training at 3 days post-injury induced a significant increase in luciferase content in spinal cord segments 3 and 4 , representing $0-4 \mathrm{~mm}$ on either side of the injury epicenter, compared to the untrained animals $(p<0.01)$. Interestingly, segments 2 and 5, representing spinal cord 4$8 \mathrm{~mm}$ from the epicenter, also had much higher levels of luciferase; however, these values were not statistically significant. Uninjured control animals demonstrated extremely low levels of luciferase extravasation, whether or not they were exposed to $8 \mathrm{~min}$ of swimming (data not shown). By 14 days post-injury, there were no differences in extravasated luciferase between swim-trained and untrained animals.

\section{Discussion}

The goal of the present study was to use our swimming model of activity-based rehabilitation (Smith et al., 2006a) to investigate if re-training applied acutely after a moderately severe thoracic SCI is more or less effective than re-training initiated at 2 weeks post-injury. At 3 days post-injury, most animals have largely recovered from the surgical trauma, but few have begun moving their hindlimbs (Multon et al., 2004); thus, it represents a feasible, acute time point to initiate training. We began with daily $2 \times 3$-min training sessions and increased the number and duration of the sessions until each animal was completing $6 \times 4$-min sessions daily starting on day 11 (Fig. 1). This is comparable to the 20-30-min daily training regimes used by a number of other groups that have initiated training acutely (Fouad et al., 2000; Hutchinson et al., 2004; Multon et al., 2004). Given that both the trained and untrained groups in the Phase 1 study showed modest improvements in swimming over time (as assessed by the LSS; Fig. 2), our approach in the Phase 2 study was to compare 

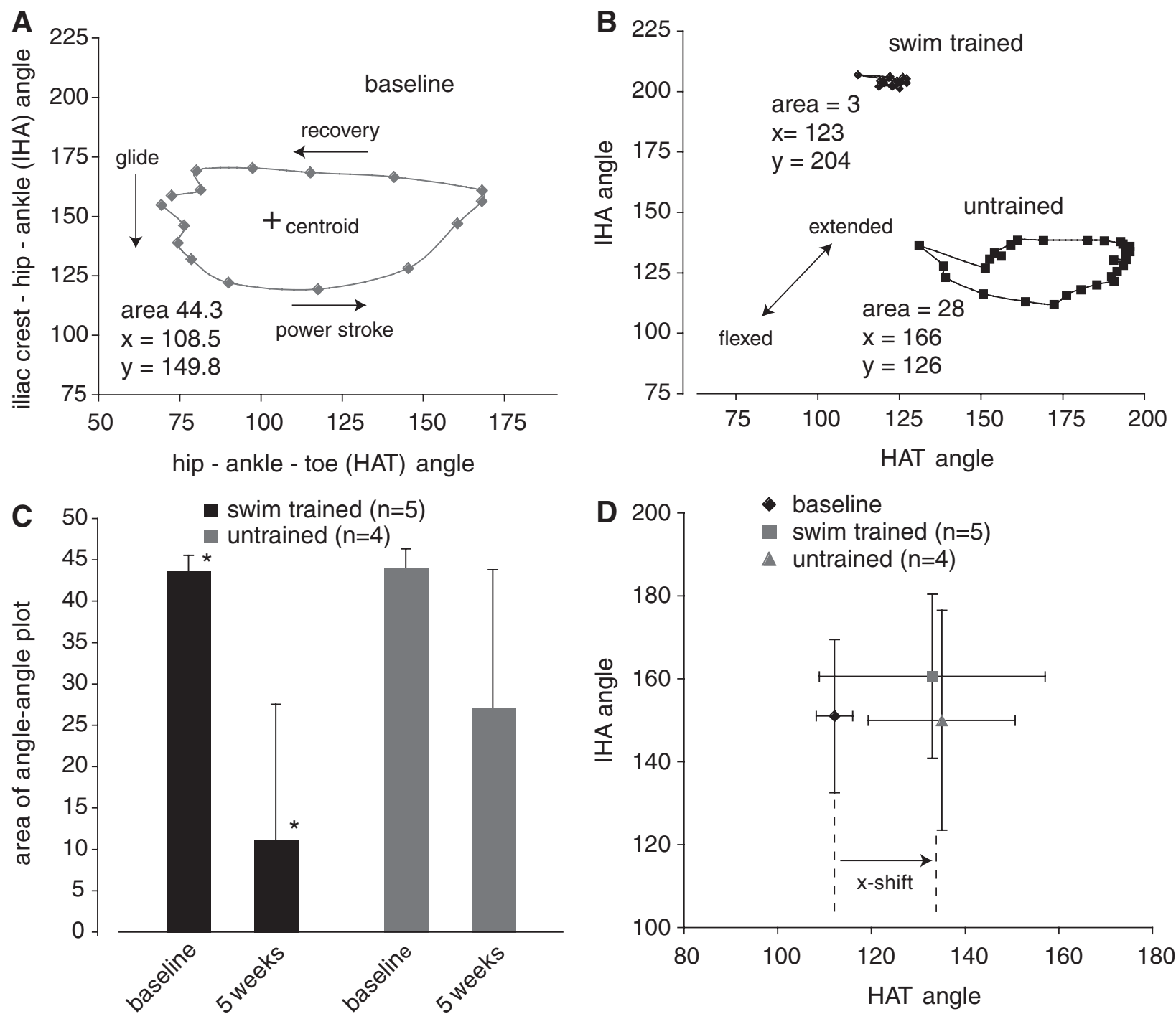

FIG. 3. (A) The angle-angle plot from a single normal swimming stroke is shown as an example. The iliac crest-hip-ankle (IHA) angle is plotted against the hip-ankle-toe (HAT) angle, with each point representing one frame of a digital video made at $60 \mathrm{~Hz}$. The main power stroke involves the extension first of the HAT ( $x$-axis), followed by extension of the IHA angle (y-axis). This is followed by a recovery stroke involving HAT flexion and period of glide with the limb flexed (glide). (B) Single strokes from a swim-trained and an untrained animal, at 5 weeks post-injury, are shown to illustrate the extremes of limb position and activity for this study. The angle-angle plot areas and centroids are shown. (C) The mean angle-angle plot areas for the trained and untrained groups are shown. The mean area for the swim-trained group was found to be significantly smaller at 5 weeks post-injury as compared to baseline (mean $\pm \mathrm{SD}, p<0.05$ ). The mean areas for the untrained animals were found to be not different from baseline (mean \pm SD). (D) The mean centroids for the angle-angle plots from both trained and untrained groups were shifted on the $x$-axis, indicating an overall more extended limb position that was not positively influenced by acute swim training with supplementary cutaneous feedback (mean \pm SD).

trained animals with a control group that was not assessed or exposed to the activity of swimming until the terminal time point. Both these approaches have drawbacks. The first appears to involve a "training effect" where 8 min of swimming each week, during the assessment of the untrained group, leads to a modest improvement in LSS scores equivalent to trained animals (Smith et al., 2006a), and the second approach involved assessing animals at 6 and 7 weeks post-injury where the behavior being assessed was novel (swimming after SCI) and the animals had not been acclimated to the apparatus or activity post-injury.
In Phase 1, both trained and untrained groups showed a modest but statistically significant improvement in LSS score over time, but no group differences were found. The mean terminal LSS score was 6, illustrating that few animals developed even occasional hindlimb movement. In contrast to our earlier results (Smith et al., 2006a) where trained animals recovered frequent hindlimb movement (LSS score of 11), the current study indicates that daily swim training starting at 3 days is no more effective than $2 \times 4$-min swimming sessions each week and that both approaches are less effective than daily swim training initiated at 2 weeks post-injury. 

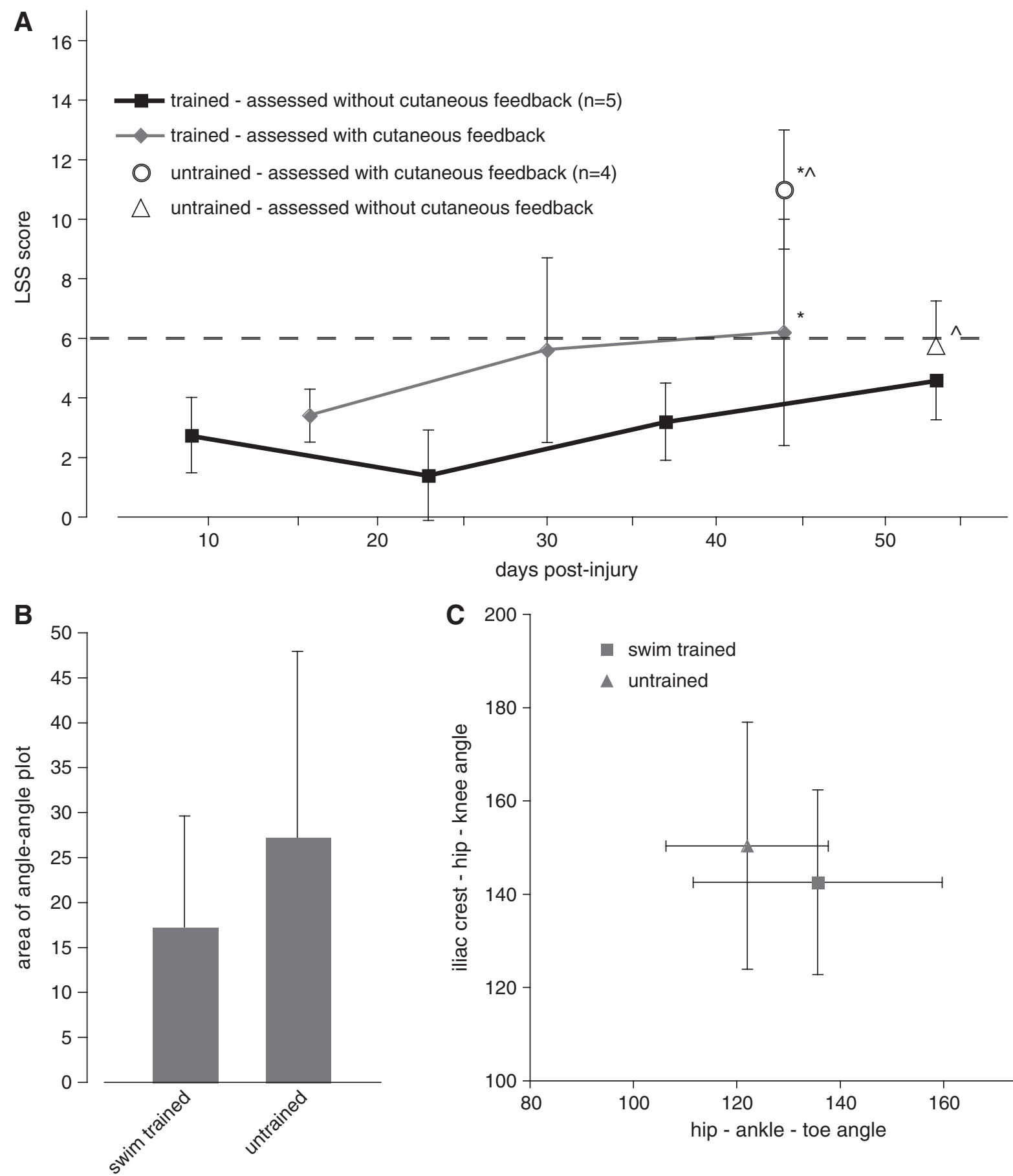

FIG. 4. (A) Shown are the Louisville Swim Scale (LSS) scores, over time, for the Phase 2 study. In red and black are the scores when assessed with or without supplementary cutaneous feedback, respectively. The open circle and triangle show the scores for the untrained group that were assessed only twice, at 44 days with supplementary cutaneous feedback and at 53 days without cutaneous feedback. The untrained group scored significantly better than the trained group at 44 days when assessed with supplementary cutaneous feedback (mean $\pm S D$, unpaired $t$-test, $p<0.05$ ), and significantly better with cutaneous feedback than without (mean $\pm \mathrm{SD}, \wedge$, paired $t$-test, $p<0.01)$. $(\mathbf{B}, \mathbf{C})$ Shown are the angle-angle plot areas and centroids, at 53 days post-injury, which were not significantly different for the trained and untrained groups.

In Phase 2, the trained group did not show significant improvements in LSS score over time; however, in agreement with the Phase 1 study, this group did achieve a score of 6 when assessed with cutaneous feedback. It is interesting to note that the mean BBB overground locomotor score for the trained group was lower, statistically, than the mean score for the untrained group. The difference in the scores was slight (1.3) but indicates that, on average, the trained animals did not achieve weight-supported stepping lacking coordination while the untrained animals did. Somewhat unexpectedly, the 

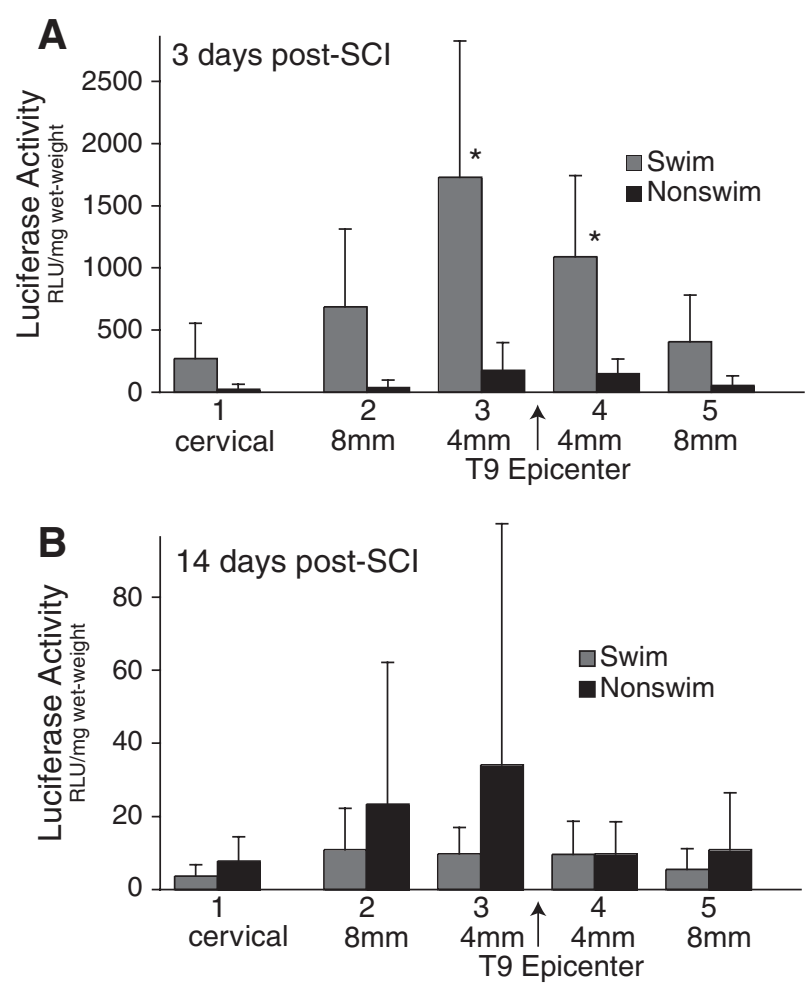

FIG. 5. Shown are the results for the luciferase assay of spinal cord tissue taken from injured rats after 8 min of swimming at 3 or 14 days post-injury. Segments of spinal cord (4 mm long) taken from the cervical enlargement (1) were compared to segments taken from just rostral and caudal to the injury epicenter. (A) Segments 3 and 4 taken from the animal that swam for $8 \mathrm{~min}$ at 3 days post-injury had significantly higher levels of luciferase activity than similar segments taken from cage-rested controls (mean $\pm S D$, $p<0.05$ ). (B) By 14 days post-injury, the levels of luciferase activity were significantly lower and were similar in cagerested and animals exposed to $8 \mathrm{~min}$ of swimming (note scale differences of the $y$-axis in $\mathrm{B}$; mean $\pm \mathrm{SD}, p<0.05$ ). Luciferase activity was below detectable limits in uninjured control animals whether or not they were exposed to $8 \mathrm{~min}$ of swimming.

untrained group in the Phase 2 study had a mean LSS score of 11 when first assessed on post-injury day 44, significantly better than the mean of 6 for the trained group and equivalent to the trained group from our standard study (Fig. 4). The untrained group also had a slightly higher (but not statistically significant) spared white matter $(13.6 \%$ compared to $11.5 \%$; Table 1). While it is unlikely that a $2 \%$ increase in mean spared white matter at the injury epicenter could account for all of these changes, it may be a contributing factor. The LSS score of 11 indicates that these animals recovered frequent hindlimb movement despite having no exposure to the activity of swimming until post-injury day 44 . This observation suggests that acute post-injury exposure to the novel, but normal locomotor activity of swimming may in some way negatively alter the plasticity of the neural circuitry involved in swimming compared to cage rest or training initiated at 2 weeks post-injury. The untrained group scored significantly lower (LSS=6; Fig. 4) when assessed without cutaneous feedback suggesting that supplementary cutaneous feedback may be more effective at encouraging hindlimb movement in animals that are untrained and/or that are not exposed to swimming with feedback until after post-injury week 2 .

Swimming kinematics cannot be accurately assessed when supplemental cutaneous feedback is being used, so all the kinematic assessments were done without cutaneous feedback. These analyses confirmed that the trained groups in both Phase 1 and 2 studies had significant deficits in hindlimb excursion after SCI, and that swim training initiated at 3 days was not able to bring about significant improvements when compared to the untrained groups (Figs. 3 and 4). Even in the absence of supplementary cutaneous feedback, as a group the untrained animals had larger, more effective kicks as shown by greater out-of-phase angular excursions. Assessment of the angle-angle plot centroid, which represents the mean hindlimb position, shows that acute swim training did not have a positive effect on limb position (Figs. 3D and 4C), which was not significantly different from the untrained groups.

As an initial effort to explore potential causes for the lack of effectiveness of acute swim re-training, we utilized an inert macromolecule, luciferase, as a surrogate to quantify the leakage of macromolecules from the blood stream into the parenchyma of the spinal cord. While a variety of surrogate markers have been used to examine the BSCB, including radiolabeled AIB (Popovich et al., 2006) and Evans Blue (Kaptanoglu et al., 2004; Benton et al., 2008), a study by Whetstone et al. (2003) demonstrated beautifully in the mouse that luciferase can be used to document the temporal changes in the BSCB following a standard thoracic moderately severe contusion injury. Their data shows that, in addition to the rapid and dramatic increase in luciferase extravasation at $24 \mathrm{~h}$ post-injury, a second peak of permeability can be observed during the 3-7-day period that includes not only the injury epicenter but also extends rostrocaudally for up to $6 \mathrm{~mm}$ (Whetstone et al., 2003). All their assessments were in cagerested animals. Our data shows that only $8 \mathrm{~min}$ of swim exercise at 3 days post-injury is sufficient to induce a significant increase in extravasated luciferase in the spinal cord, on either side of the injury epicenter, compared to similar segments taken from animals that were returned to their cages without exercise after receiving the intravenous injections of luciferase. The $0-4$-mm segments (i.e., those immediately adjacent to the injury epicenter) showed the greatest increases in luciferase, while the rostral and caudal segments $4-8 \mathrm{~mm}$ from the epicenter showed substantial increases that were not statistically significant because of variability. Interestingly, huge differences in luciferase activity were also seen when comparing swim exercise at 3 and 14 days post-injury, suggesting that the BSCB had largely recovered by that time (Popovich et al., 2006), or that swimming at 14 days was less effective at bringing about an increase in blood pressure and/or flow. When uninjured rats received $8 \mathrm{~min}$ of swim exercise the levels of luciferase were undetectable. It is important to note that the swimming sessions essentially exhausted the animals. While scheduled for 4 min each, many of the 3-day animals were not able to complete the sessions without significant assistance. Nonetheless, the activity was brief and the animals appeared to completely recover from each session within a few minutes, and returned to grooming or sleeping soon after being placed back in their home cages.

In addition to the goal of improving overground locomotion (mobility), activity-based rehabilitation approaches 
have also been shown to influence a number of systems, including pain (Hutchinson et al., 2004), bone and muscle wasting (de Bruin et al., 1999; Giagregoria et al., 2005; Liu et al., 2008), spasticity (Fung et al., 1990), and cardiovascular/ respiratory function (Ditor et al., 2005). Thought to be common to all these studies is the involvement, either directly or indirectly, of trophic and/or growth factors, which respond to activity and exercise in both intact animals and during post-injury rehabilitation (Gomez-Pinilla et al., 2002). However, increases in physical activity (exercise) require variable increases in heart rate and blood flow, depending on the level of activity. When initiated acutely, these increases occur at a time when the bloodbrain barrier/BSCB is compromised (Popovich et al., 1997), potentially leading to exacerbation of inflammatory processes in and around the injury site (Griesbach et al., 2004). Thus, while activity-based therapeutic approaches initiated acutely may benefit functional recovery via growth factor production and other sources of neural plasticity, they may be detrimental to functional recovery due to exacerbation of the acute-phase inflammatory processes. Importantly, it has been demonstrated that the temporal aspects of the inflammatory cascade following contusive spinal cord injuries in the human population are fairly similar to those described in animal models (Fleming et al., 2006).

Previous studies that have utilized acute re-training strategies following incomplete spinal cord injuries have relied on treadmill step training (Fouad et al., 2000; Multon et al., 2004) and assessments of overground stepping as primary outcome measures. While Fouad et al. (2000) reported no improvements in overground stepping, grid-walking, or narrowbeam walking in animals with dorsal hemisections, Multon et al. (2004) reported modest improvements in stepping with training following thoracic contusion injuries. While treadmill training involves some handling and stress for the animals, these authors make no comment about the activity being physically tiring for the animals. It remains to be determined what levels of physical activity or exercise may be counter-productive during the acute and sub-acute postinjury time points and whether or not the detrimental influence is directly related to exercise-induced increases in heart rate and blood flow or if the release of stress-related compounds may also play a role.

In summary, these studies demonstrate that swim training, initiated at 3 days post-SCI is less effective at bringing about task specific improvements in swimming than when training is initiated at 2 weeks. Furthermore, our data suggest that swimming, and perhaps any "forced exercise" that can significantly increase the cardiovascular output at acute or sub-acute post-SCI time points, can bring about increased extravasation of macromolecules into the spinal cord parenchyma, potentially altering the progression of the secondary injury. To our knowledge, this is the first demonstration of activity-dependent microvascular dysfunction following traumatic SCI. Further, these findings imply that secondary injury cascades may be directly influenced by even short events leading to increases in blood pressure during the acute and sub-acute time periods. Events that lead to even shortlived peaks in cardiac output, as might be expected during routine treatment and the initialization of rehabilitation efforts, could have long-term negative consequences on functional outcome.

\section{Acknowledgments}

We would like to acknowledge the excellent technical assistance of Melissa Maddie, Christine Nunn, Kim Fentress, Aaron Puckett, Johnny Morehouse, and Victoria Druzhinina. These studies were supported by grants from the Kentucky Spinal Cord and Head Injury Research Trust and the NIH (R01 NS052292, P20 RR015576, P20 RR015576).

\section{Author Disclosure Statement}

No competing financial interests exist.

\section{References}

Abel, R., Schablowski, M., Rupp, R., and Gerner, H.J. (2002). Gait analysis on the treadmill-monitoring exercise in the treatment of paraplegia. Spinal Cord 40, 17-22.

Basso, D.M., Beattie, M.S., and Bresnahan, J.C. (1995). A sensitive and reliable locomotor rating scale for open field testing in rats. J. Neurotrauma 12, 1-21.

Benton, R.L., Maddie, M.A., Minnillo, D.R., Hagg, T., and Whittemore, S.R. (2008). Griffonia simplicifolia isolectin B4 identifies a specific subpopulation of angiogenic blood vessels following contusive spinal cord injury in the adult mouse. J. Comp. Neurol. 507, 1031-1052.

Boyce, V., Tumolo, M., Fischer, I., Murray, M., and Lemay, M. (2007). Neurotrophic factors promote and enhance locomotor recovery in untrained spinalized cats. J. Neurophysiol. 98, 1988-1996.

de Bruin, E.D., Frey-Rindova, P., Herzog, R.E., Dietz, V., Dambacher, M.A., and Stussi, E. 1999. Changes of tibia bone properties after spinal cord injury: effects of early intervention. Arch. Phys. Med. Rehabil. 80, 214-220.

de Leon, R., Hodgson, J., Roy, R.R., and Edgerton, V.R. (1998). Locomotor capacity attributable to step training versus spontaneous recovery after spinalization in adult cats. J. Neurophysiol. 79, 1329-1340.

Ditor, D.S., Kamath, M.V., MacDonald, M.J., Bugaresti, J., McCartney, N., and Hicks, A.L. 2005. Effects of body weightsupported treadmill training on heart rate variability and blood pressure variability in individuals with spinal cord injury. J. Appl. Physiol. 98, 1519-1525.

Dupont-Versteegden, E.E., Houle, J.D., Dennis, R.A., Zhang, J., Knox, M., Wagoner, G., Peterson, C.A. (2004). Exerciseinduced gene expression in soleus muscle is dependent on time after spinal cord injury in rats. Muscle Nerve 29, 73-81.

Engesser-Cesar, C., Ichiyama, R.M., Nefas, A.L., Hill, M.A., Edgerton, V.R., Cotman, C.W., and Anderson, A.J. (2007). Wheel running following spinal cord injury improves locomotor recovery and stimulates serotonergic fiber growth. Eur. J. Neurosci. 25, 1931-1939.

Ferson, S., Rohlf, F.J., and Koehn, R.K. (1985). Measuring shape variation of two-dimensional outlines. Syst. Zool. 34, 59-68.

Felici, F., Bernardi, M., Rodio, A., Marchettoni, P., and Macaluso, A. (1997). Rehabilitation of walking for paraplegic patients by means of a treadmill. Spinal Cord 35, 383-385.

Fleming, J.C., Norenberg, M.D., Ramsay Dam Dekaban, G.A., Marcillo, A.E., Saenz, A.D., Pasquale-Styles, M., Dietrich, W.D., and Weaver, L.C. (2006). The cellular inflammatory response in human spinal cords after injury. Brain 129, 32493269.

Fung, J., Stewart, J.E., and Barbeau, H. (1990). The combined effects of clonidine and cyproheptadine with interactive training on the modulation of locomotion in spinal cord injured subjects. J. Neurol. Sci. 100, 85-93. 
Gazzani, F., Bernardi, M., Macaluso, A., Coratella, D., Ditunno, Jr., J., Castellano, V., Torre, M., Macellari, V., and Marchetti, M. (1999). Ambulation training of neurologic patients on the treadmill with a new Walking Assistance and Rehabilitation Device (WARD). Spinal Cord 37, 336-344.

Giangregorio, L.M., Hicks, A.L., Webber, C.E., Phillips, S.M., Craven, B.C., Bugaresti, J.M., and McCartney, N. (2005). Body weight supported treadmill training in acute spinal cord injury: impact on muscle and bone. Spinal Cord 43, 649-657.

Gomes-Leal, W., Corkill, D.J., and Picanco-Diniz, C.W. (2005). Systemic analysis of axonal damage and inflammatory response in different white matter tracts of acutely injured rat spinal cord. Brain Res. 1066, 57-70.

Gomez-Pinilla, F., So, V., and Kesslak, J. (1998). Spatial learning and physical activity contribute to the induction of fibroblast growth factor: neural substrates for increased cognition associated with exercise. Neuroscience 85, 53-61.

Gomez-Pinilla, F., Ying, Z., Opazo, P., Roy, R.R., and Edgerton, V.R. (2001). Differential regulation by exercise of BDNF and NT-3 in rat spinal cord and skeletal muscle. Eur. J. Neurosci. $13,1078-1084$.

Gomez-Pinilla, F., Ying, Z., Roy, R.R., Molteni, R., and Edgerton, V.R. (2002). Voluntary exercise induces a BDNF-mediated mechanism that promotes neuroplasticity. J. Neurophysiol. 88, 2187-2195.

Griesbach, G.S., Gomez-Pinilla, F., and Hovda, D.A. (2004). The upregulation of plasticity-related proteins following TBI is disrupted with acute voluntary exercise. Brain Res. 1016, 154-162.

Hagg, T., and Oudega, M. (2006). Degenerative and spontaneous regenerative processes after spinal cord injury. J. Neurotrauma 23, 264-280.

Harkema, S., Hurley, S., Patel, U., Requejo, P., Dobkin, B., and Edgerton, V.R. (1997). Human lumbosacral spinal cord interprets loading during stepping. J. Neurophysiol. 77, 797-811.

Hausmann, O.N. (2003). Post-traumatic inflammation following spinal cord injury. Spinal Cord 41, 369-378.

Hays, W. (1981). Statistics, $3^{\text {rd }}$ ed. Holt, Rinehart and Winston: New York.

Hutchinson, K., Gomez-Pinilla, F., Crowe, M., Ying, Z., and Basso, D.M. (2004). Three exercise paradigms differentially improve sensory recovery after spinal cord contusion in rats. Brain 127, 1403-1414.

Kaptanoglu, E., Okutan, O., Akbiyik, F., Solaroglu, I., Kilinc, A., and Beskonakli, E. (2004). Correlation of injury severity and tissue Evans blue content, lipid peroxidation and clinical evaluation in acute spinal cord injury in rats. J. Clin. Neurosci. 11, 879-885.

Karimi-Abdolrezaee, S., Eftekhar, E., Wang, J., Morshead, C., and Fehlings, M. (2006). Delayed transplantation of adult neural precursor cells promotes remyelination and functional neurological recovery after spinal cord injury. J. Neurosci. 26, 3377-3389.

Liu, M., Bose, P., Walter, G.A., Thompson, F.J., and Vandenborne, K. (2008). A longitudinal study of skeletal muscle following spinal cord injury and locomotor training. Spinal Cord 46, 488-493.

Loy, D.N., Crawford, C.H., Darnall, J.B., Burke, D.A., Onifer, S.M., and Whittemore, S.R. (2002). Temporal progression of angiogenesis and basal lamina deposition after contusive spinal cord injury in the adult rat. J. Comp. Neurol. 445, 308-324.

Magnuson, D.S.K., Smith, R.R., Brown, E.H., Enzmann, G., Angeli, C., Quesada, P.M., and Burke, D. (2009). Swimming as a model of task-specific locomotor re-training after spinal cord injury in the rat. Neurorehab. Neural Rep. (in press; DOI: $10.1177 / 1545968308331147)$.

Maegele, M., Muller, S., Wernig, A., Edgerton, V.R., and Harkema, S. (2002). Recruitment of spinal motor pools during voluntary movements versus stepping after human spinal cord injury. J. Neurotrauma 19, 1217-1229.

Merrill, J.E., Ignarro, J.M., Sherman, P., Melinek, J., and Lane, T.E. (1993). Microglial cell cytotoxicity of oligodendrocytes is mediated through nitric oxide. J. Immunol. 151, 2132-2141.

Muir, G.D., and Steeves, J.D. (1995). Phasic cutaneous input facilitates locomotor recovery after incomplete spinal cord injury in the chick. J. Neurophysiol. 74, 358-368.

Multon, S., Franzen, R., Poirrier, A.-L., Scholtes, F., and Schoenen, J. (2003). The effect of treadmill training on motor recovery after a partial spinal cord compression- injury in the adult rat. J. Neurotrauma 20,699-706.

Pepin, A., Norman, K.E., and Barbeau, H. (2003). Treadmill walking in incomplete spinal-cord-injured subjects: 1. Adaptation to changes in speed. Spinal Cord 41, 257-270.

Popovich, P., Wei, P., and Stokes, B. (1997). Cellular inflammatory response after spinal cord injury in sprague-dawley and lewis rats. J. Comp. Neurol. 377, 443-464.

Rabchevsky, A.G., and Streit, W.J. (1998). Role of microglia in postinjury repair and regeneration of the CNS. Ment. Retard. Dev. Disabil. Res. 4, 187-192.

Rossignol, S. (2002). Locomotion and its recovery after spinal cord injury in animal models. Neurorehab. Neural Rep. 16, 201-205.

Schnell, L., Fearn, S., Klassen, H., Schwab, M., and Perry, H. (1999). Acute inflammatory responses to mechanical lesions in the CNS: differences between brain and spinal cord. Eur. J. Neurosci. 11, 3648-3658.

Schnell, L., Fearn, S., Klassen, H., Schwab, M., and Perry, H. (2006). Acute inflammatory responses to mechanical lesions in the CNS: differences between brain and spinal cord. Eur. J. Neurosci. 11, 3648-3658.

Scivoletto, G., Morganti, B., and Molinari, M. (2005). Early versus delayed inpatient spinal cord injury rehabilitation: an Italian study. Arch. Phys. Rehabil. 86, 512-516.

Smith, R., Burke, D., Baldini, A., Shum-Siu, A., Baltzley, R., Bunger, M., and Magnuson, D. (2006b). The Louisville Swim Scale: a novel assessment of hindlimb function following spinal cord injury in adult rats. J. Neurotrauma 23, 1654-1670.

Smith, R., Shum-Siu, A., Baltzley, R., Bunger, M., Baldini, A., Burke, D., and Magnuson, D. (2006a). Effects of swimming on functional recovery after incomplete spinal cord injury in rats. J. Neurotrauma 23, 908-918.

Whetstone, W.D., Hsu, J.-Y.C., Eisenberg, M., Werb, Z., and Noble-Haeusslein, L.J. (2003). Blood-spinal cord barrier after spinal cord injury: relation to revascularization and wound healing. J. Neurosci. Res. 74, 227-239.

Ying, Z., Roy, R.R., Edgerton, V.R., and Gomez-Pinilla, F. (2005). Exercise restores levels of neurotrophins and synaptic plasticity following spinal cord injury. Exp. Neurol. 193, 411419 .

Address correspondence to: David S.K. Magnuson, Ph.D. Kentucky Spinal Cord Injury Research Center Department of Neurological Surgery University of Louisville School of Medicine 511 South Floyd Street, MDR 616 Louisville, KY 40202

E-mail: dsmagn01@louisville.edu 
